\title{
Long-Lasting Effect of Changes in Incubation Temperature on Heat Stress Induced Neuronal Hypothalamic c-Fos Expression in Chickens
}

\author{
Oliver Janke and Barbara Tzschentke*
}

\author{
Humboldt-University of Berlin, Institute of Biology, Working Group Perinatal Adaptation, Philippstrasse 13, 10115 \\ Berlin, Germany
}

\begin{abstract}
In birds, during prenatal 'critical periods' the thermoregulatory system can be life-long imprinted by the actual incubation temperature. The aim of the present study is to elucidate long-term effects of prenatal temperature experiences, applied at the end of incubation, on central thermoregulatory mechanisms by detection of neuronal hypothalamic c-Fos expression as a consequence of acute heat stress in growing and adult chickens using immunohistochemistry. From day 18 of incubation chicken embryos were incubated in three temperature groups: $37.5^{\circ} \mathrm{C}$ (regular incubated), $34.5^{\circ} \mathrm{C}$ (cold incubated) or $38.5^{\circ} \mathrm{C}$ (warm incubated). C-Fos expression was detected in 29 4-weeks old and 618 -weeks old chickens after acute heat stress $\left(42^{\circ} \mathrm{C}\right.$ for $\left.90 \mathrm{~min}\right)$. The results show, that prenatal temperature experiences can induce long-lasting changes in the heat induced neuronal hypothalamic c-Fos expression. But significant alterations could be only found in 8 weeks old chickens and these changes were in opposite as expected. It means that after acute heat stress cold incubated chickens have a significantly lower neuronal c-Fos expression compared with the warm incubated ones. This effect could be caused by cross adaptation to the actual ambient temperature during the growing period. Further, the developmental pattern of the hypothalamic neuronal network in different incubated birds, which is demonstrated by neuronal c-Fos expression in the actual study, is similar to the pattern, which we found after recordings of single neurons activity, to a higher extent. Correlations between both parameters, which are involved in neuronal plasticity, can be accepted.
\end{abstract}

Keywords: Hypothalamus, neuronal c-Fos expression, thermoregulation, incubation temperature, imprinting.

\section{INTRODUCTION}

During 'critical periods' in the prenatal as well as early postnatal ontogeny, the actual environment influences the development of the respective physiological control systems for the entire life period. In birds, the thermoregulatory system can be life-long imprinted by the actual incubation temperature [1], especially at the end of incubation. The final incubation period in precocial species is characterized by well-developed peripheral and central nervous thermoregulatory mechanisms, and body functions $[2,3]$ as well as dramatic changes in the quality of regulatory processes [1]. Most functional systems develop from open loop systems without feedback control into closed control systems regulated by feedback mechanisms. It is a 'critical period' in the development of body functions. In precocial poultry species, for instance, at the end of incubation, long-term alterations in incubation temperature may induce prenatal epigenetic temperature adaptation, which results in a longlasting cold or warm adaptation during post hatching development. Minne and Decuypere [4] found that cold incubated chicken had a higher heat production over a range of ambient temperatures for up to 8 weeks of post-hatching. The higher heat production was accompanied with higher $\mathrm{T} 3$ and $\mathrm{T} 4$ levels. On the first day of post hatching Muscovy ducklings incubated at lower temperatures than normal have a $56 \%$ higher heat production and a higher deep body temperature

*Address correspondence to this author at the Humboldt-University of Berlin, Institute of Biology, Working Group Perinatal Adaptation, Philippstrasse 13, 10115 Berlin, Germany; Tel: +49 302093 6276;

Fax: +49 302093 6008; E-mail: barbara.tzschentke@rz.hu-berlin.de under cold load $\left(1\right.$ hour at $\left.10^{\circ} \mathrm{C}\right)$ as compared to controls. Cold incubated birds are able to control their actual deep body temperature at this set-point, in contrary to those incubated at $37.5^{\circ} \mathrm{C}$, which display a lower heat production. In a temperature gradient, Muscovy ducklings incubated at a low temperature preferred a significantly lower temperature than birds incubated at the normal incubation temperature during the first 10 days of post hatching. This supports the hypothesis that avian prenatal cold experience leads to a downward shift of the thermoregulatory set-point [5]. On the other hand, the preferred ambient temperature in 1- to 10days-old turkeys is higher after a prenatal heat load $\left(38.5^{\circ} \mathrm{C}\right)$ than in birds incubated at the normal temperature $\left(37.5^{\circ} \mathrm{C}\right)$. This indicates an elevation of the thermoregulatory set-point after prenatal heat load [5]. Changes in the thermosensitivity of the control centre of the thermoregulatory system reflect the changes in peripheral thermoregulatory mechanisms after prenatal temperature experiences. In Muscovy ducklings, during the first days of life changes in incubation temperature induced a clear alteration of neuronal hypothalamic thermosensitivity [6]. On the $10^{\text {th }}$ day of post-hatching it could be observed that prenatal cold load elevated the neuronal hypothalamic warm-sensitivity, with an increased proportion of warm and a reduced proportion of cold sensitive neurones in comparison with the control group. Prenatal warm load induced the opposite effect. Within a few weeks after hatching these shifts disappeared probably due to the interference of acclimation to the actual postnatal environment and the reorganization of the neuronal network involved in thermoregulation (unpublished own results). CFos is expected to be a more sensible marker to show central nervous temperature adaptation for a longer period in birds 
as had been previously demonstrated in rats by Miyata and co-worker [7]. Cold exposure of infant rats changed c-Fos expression to acute cold stimulation in hypothalamic brain regions of the adults. The newborn rats were exposed until the age of 14 days for $2-4 \mathrm{~h}$ at $4^{\circ} \mathrm{C}$ every day. A cold stimulus of $10^{\circ} \mathrm{C}$ for $3 \mathrm{~h}$ in the $15^{\text {th }}$ week evoked significantly lower neuronal c-Fos expression when compared to the control particularly in the hypothalamus. C-Fos is a nuclear phosphoprotein coded by the proto-oncogen c-fos, which is one of the immediate-early genes (IEG). The protein c-Fos dimerises with the Jun protein to form the transcription factor AP-1. AP-1 binds to different DNA-sites and thus influences the transcription rate of late effector genes [8]. The expression of the IEG product c-Fos is induced by a variety of stimuli, like social and environmental factors as well as after different forms of learning. In piglets, a single exposure to social stress already elevated c-fos mRNA expression in the hypothalamus and amygdala [9]. Acute heat load, for instance, induced strong c-Fos expression in hypothalamic neurons in mice and rats $[10,11]$, short-term after application. After osmotic stress, c-Fos expression increased in salt gland cells of ducks [12]. Because of it, cFos expression is often used as an unspecific stress marker. Further, c-Fos is involved in long-term modifications in neuronal biochemistry and structure proposed to underlie learning and memory $[13,14,15,16,17,18]$, which finally characterize neuronal plasticity. The necessity of c-Fos expression for longer-term memory was also shown in poultry chicks $[19,20,21]$.

Compared to single cell recordings, the c-Fos method offers a valuable tool for morphological identification of numerous activated neurons at one time and thus demonstrates neuronal networks. In the nucleus of the cell, c-Fos can be detected by immunohistochemical method.

The aim of the present study is to elucidate long-term effects of prenatal temperature experiences, applied at the end of incubation, on central thermoregulatory mechanisms by detection of neuronal hypothalamic c-Fos expression as a consequence of acute heat stress in growing and adult chickens using immunohistochemistry.

\section{MATERIALS AND METHODS}

\section{Incubation and Keeping of Chickens}

In the present study 4 weeks $(n=35)$ and 8 weeks $(n=$ 68) old White Leghorn chickens were investigated. Lohmann Tierzucht $\mathrm{GmbH}$, Cuxhaven, Germany, supplied the eggs. The embryos were incubated at $37.5^{\circ} \mathrm{C}, 70 \%$ relative humidity, and continuously turned in horizontal position. From day 18 of incubation onwards eggs were not turned and separated into three incubation groups. Until hatching, the one group was incubated at the regular $37.5^{\circ} \mathrm{C}$ and two groups of embryos were exposed to altered constant incubation temperatures viz. cold $\left(34.5^{\circ} \mathrm{C}\right)$ and warm $\left(38.5^{\circ} \mathrm{C}\right)$ with a relative humidity of $90 \%$. Because day 3 of post-hatching is a critical period for the development of the thermoregulatory system too [22], the chickens of each incubation group were kept at different temperatures, which continue the chronic temperature load during embryonic development. Until day 3 of post-hatching the cold incubated group was kept at $30^{\circ} \mathrm{C}$, the warm incubated at $34.5^{\circ} \mathrm{C}$ and the control group at $33^{\circ} \mathrm{C}$ (usual temperature regime). From day 3 until the experimental age of 4 or 8 weeks the birds were kept under the usual temperature conditions for chickens regarding to the recommendations of the European Community. Food (standard food for chicken of different age groups, ssniff Spezialdiäten, Germany) and water were available ad libitum.

\section{Experimental Procedure}

Prior to the immunohistochemical investigations at the age of 4 or 8 weeks, respectively, acute heat stress $\left(42^{\circ} \mathrm{C}\right)$ for 90 min was applied to the chickens of all incubation groups. Temperature and duration of the application were determined in previous experiments [23]. For control of the influence of acute heat stress on neuronal c-Fos expression, in some chickens of each tested age group the c-Fos expression was determined without application of heat stress (control).

All animal experiments were done according to approved protocols, in compliance with the German Protection of Animals Act and with permission of the Berlin administration (Reg. 0209/05).

\section{Immunohistochemistry}

The chickens were anaesthetized and transcardial perfusion was performed with $50 \mathrm{ml}$ phosphate-buffered saline (pH 7.4) and $150 \mathrm{ml}$ of $4 \%$ paraformaldehyde in phosphate buffer (PB). After perfusion, brains were dissected and stored for an hour in the paraformaldehyde solution. Later they were transferred into $20 \%$ sucrose in PB and left overnight at $4^{\circ} \mathrm{C} .20 \mu \mathrm{m}$ brain sections were made using a cryostate (Leica Microsystems, Wetzlar). Sections containing the hypothalamic region were attached to poly-L-Lysine coated micro slides. After washing in PB they were stored for one hour in a blocking solution containing $10 \%$ normal horse serum and $0.3 \%$ Triton X-100 (Sigma) in PB. Then the primary antibody (anti chicken c-Fos, made in rabbit; Santa Cruz Biotechnology, Heidelberg) was incubated with the sections at $4^{\circ} \mathrm{C}$ for $48 \mathrm{~h}$ at a concentration of $1: 1000$. After washing with $\mathrm{PB}$ a biotinylated anti rabbit $\mathrm{IgG}$ (Axxora Deutschland $\mathrm{GmbH}$, Grünberg) 1:200 in PB was bound to the previously formed complex during two hours incubation at room temperature. This incubation was followed by a second blocking step $\left(0.3 \% \mathrm{H}_{2} \mathrm{O}_{2}\right.$ in methanol for $\left.25 \mathrm{~min}\right)$ and washing in PB. By application of an ABC-solution (Vectastain Elite ABC Kit, Axxora Deutschland $\mathrm{GmbH}$, Grünberg) horseradish peroxidase was attached to the immune complex. The latter served to bind diaminobenzidine tetrahydrochloride (DAB) $50 \mathrm{mg} / 100 \mathrm{ml}$ (Sigma) in the presence of $0.0001 \% \mathrm{H}_{2} \mathrm{O}_{2}$ in the next step. DAB evoked a brown staining of the c-Fos positive cell nuclei of neurons. Later the sections were counter-stained using cresylviolet for visualization of the brain structures. Control sections were treated the same way without incubation with the primary antibody. Finally tissue sections were covered with Entellan (Merck) and glass coverslips.

\section{Analysis}

For analysis light microscopy and digital photography at a magnification of 50 fold (Zeiss Axioskop II, Zeiss AxioCam HRc) were used. C-Fos positive neurons were 


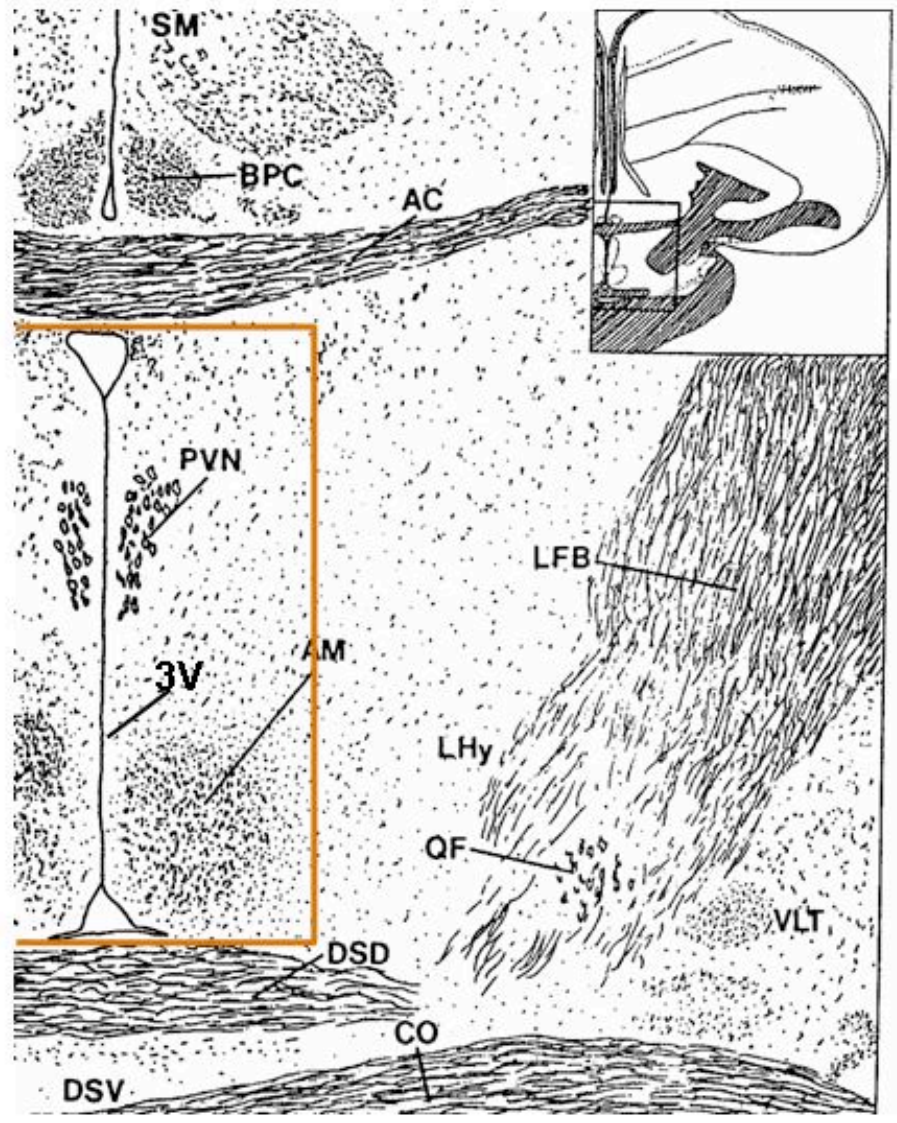

Fig. (1). Schematic picture of the PO/AH after Kuenzel and van Tienhoven [25]. The area between the commissura anterior (AC) and the chiasma opticum (CO) were c-Fos positive neurons were counted is marked. AM: Nucleus anterior medialis hypothalami PVN: nucleus paraventricularis, $3 \mathrm{~V}$ : third ventricle

counted in the preoptic area of the anterior hypothalamus $(\mathrm{PO} / \mathrm{AH})$. Stereotaxic data of the adult chicken brain were taken from [24]. Fig. (1) shows a schematic picture of the PO/AH after Kuenzel and van Tienhoven [25]. The area were c-Fos positive neurons were counted is marked (1500 $\mu \mathrm{m}$ left and right from $3^{\text {rd }}$ ventricle between commissura anterior and chiasma opticum). An example of c-Fos positive neurons in the chicken PO/AH is given in Fig. (2). Means of three successive brain sections of each chicken were calculated for further analysis. The intensity of c-Fos expression between chickens of the three incubation groups was compared with application of the t-test $+\alpha$-correction, because the values were normal distributed. Normal distribution was tested using the Kolmogoroff-SmirnoffTest. The level of significance was $\alpha=0.05$.

\section{RESULTS}

In Table 1 the numbers of chickens investigated in each experimental group are presented. In control chickens without acute heat stress only few neurons expressing c-Fos were found in the PO/AH (Fig. 3). Control sections, which were made without incubation with the primary antibody, did not show DAB staining in the nuclei. After acute heat exposure in all differently incubated embryos c-Fos was expressed in a high number of neurons. Among the incubation groups in 4 weeks old chickens the means of c-
Fos positive neurons in the $\mathrm{PO} / \mathrm{AH}$ were not significantly different at the significance level of $\alpha=0.05$. Whereas, in 8 weeks old chickens prenatal temperature experience induced a significantly lower heat stress shown by neuronal c-Fos expression in the cold incubated group compared with the warm incubated one (Fig. 3).

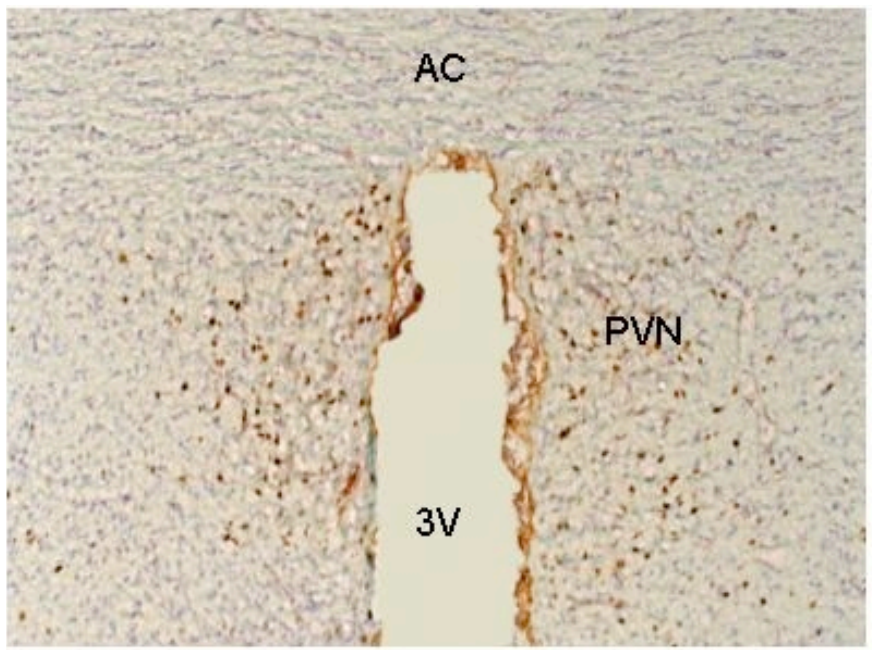

Fig. (2). Example of c-Fos positive neurons (dark brown) in the chicken $\mathrm{PO} / \mathrm{AH}$ (magnification: 50 fold). AC: commissura anterior, $\mathrm{PVN}$ : nucleus paraventricularis, $3 \mathrm{~V}$ : third ventricle. 


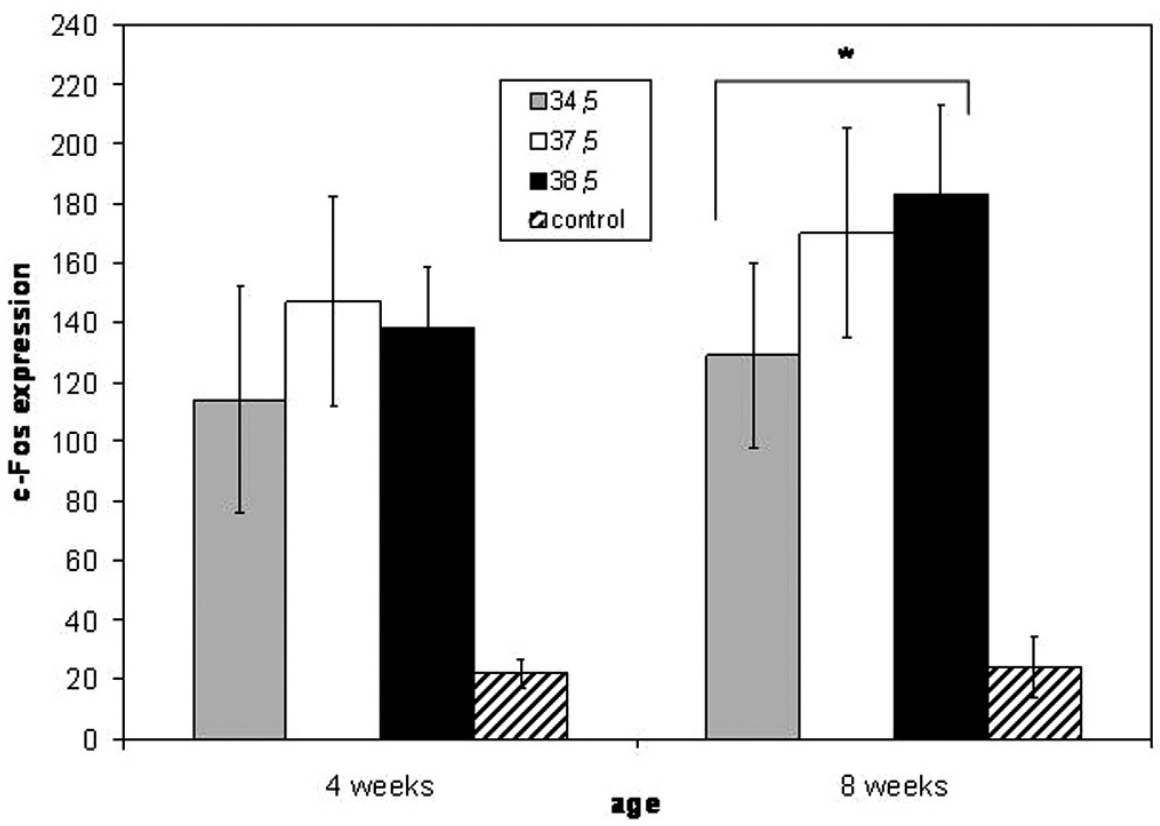

Fig. (3). C-Fos-positive PO/AH neurons in 4 and 8 weeks old chickens incubated under different temperatures during the last 4 days of incubation. Asterisk represents statistically significantly differences between the different incubated chickens at the level of $p<0.05$. The control group includes chickens without acute heat stress prior to the immunohistocemical investigations (statistically significantly different to all heat stressed groups, $\mathrm{p}<0.001$ ).

Table 1. Numbers of Investigated 4- and 8 Weeks Old Chickens of Different Experimental Groups

\begin{tabular}{|c|c|c|}
\hline Incubation temperature & $\begin{array}{c}\text { 4 weeks old } \\
\text { chickens }\end{array}$ & $\begin{array}{c}\text { 8 weeks old } \\
\text { chickens }\end{array}$ \\
\hline \hline With acute heat stress & 10 & 20 \\
34.5 & 6 & 23 \\
37.5 & 13 & 18 \\
38.5 & 6 & 7 \\
\hline Without acute heat stress & 35 & 68 \\
\hline $34.5,37.5,38.5$ & & \\
\hline Total & & \\
\hline
\end{tabular}

\section{DISCUSSION AND CONCLUSION}

The results show, that prenatal temperature experiences can induce long-lasting changes in the neuronal hypothalamic c-Fos expression after acute heat stress. But significant alterations could be found only in 8 weeks old chickens and these changes were in opposite as expected. If an increased incubation temperature during the last 4 days of embryonic development induced a long-lasting warm adaptation, acute heat load in the adult birds should cause a lower neuronal cFos expression in the regulatory brain area in comparison with regular and cold incubated birds. The opposite effect was found in our actual study. We assume that this effect is caused by cross adaptation to the actual ambient temperature during the growing period. After day 3 of post-hatching chicken of all incubation groups were kept at similar temperatures.
However, the significant differences in neuronal c-Fos expression between cold and warm incubated adult chickens are a sign for long-lasting changes in the neuronal network controlling body temperature. Induced by changes in neuronal activity c-Fos is involved in neuronal plasticity, that means changes in synaptic contacts [26, 27], and changes in effector gene expression possibly by epigenetic mechanisms. Changes in neuronal gene expression as well as in epigenetic control of gene regulation were found in the chicken hypothalamus after thermal (warm) conditioning exclusively on day 3 of post-hatching, which is also critical for thermal control establishment. Clear alterations occurred, for instance, in the R-Ras-3 gene expression [28], in brainderived neurotrophic factor [29] as well as in DNA and histone methylation of different genes [30-32].

In our previous electrophysiological investigations $[2,6$, 33-36] we found long-lasting changes in hypothalamic neuronal activity after prenatal temperature experiences. These long-lasting reorganizations in the neuronal network controlling body temperature, induced by prenatal warm or cold incubation but also under normal incubation conditions, occur stepwise during different developmental periods. In normal incubated birds a stepwise development of the neuronal hypothalamic thermosensitivity occurs during the early post hatching ontogeny. In Muscovy duck embryos as well as during the first days of post hatching in Muscovy ducklings and chickens the neuronal hypothalamic thermosensitivity is characterized by a high cold-sensitivity $[33,36]$. Around day 10 in Muscovy ducklings [33] and day 30 in chickens [36], respectively, the neuronal hypothalamic thermosensitivity is changing from the juvenile into the adult type, which is in birds as well as mammals characterized by a higher warm sensitivity [37]. 
In Muscovy ducks prenatal warm or cold load during the last days of incubation altered the neuronal hypothalamic thermosensitivity at the last day of incubation already [34]. But the changes were not significant and independent of the temperature experienced within the prenatal period (proximate non-adaptive). It means, for instance, that the proportion in warm and cold sensitive neurons was increasing, both, when incubation temperature was increased or decreased from the control level. On day 1 of post-hatching, this trend was continuing and becomes significant in the proportion of cold sensitive neurons [6]. On day 5 of posthatching these alterations were also significant in the proportion of warm sensitive neurons, but in cold sensitive ones a development into the opposite direction was found [6].

In the course of embryonic development, stimulation of body functions due to changes in the environmental conditions induces as a rule first uncoordinated and immediate (proximate) non-adaptive reactions [35]. It seems that during the early development of body functions it is not important for the organism that an adaptable reaction occurs but rather the fact that a reaction occurs anyway is important for the adaptability during later life. At the end of embryonic or during the early postnatal period a qualitative change occurs in the reaction pattern of body function after environmental stimulation. The uncoordinated and/or immediate non-adaptive "training" reactions change into coordinated and/or adaptive reactions [35]. In Muscovy ducks such a new qualitative level was reached on day 10 of life; the changes were proximate adaptive [6]. It means that prenatal cold experience increases the neuronal hypothalamic warm sensitivity and prenatal heat experience increases neuronal hypothalamic cold sensitivity. But these alterations disappeared in 30 days old Muscovy ducks (non published results). In these age group no significant differences in the neuronal thermosensitivity were found between the different incubated birds; which could be a result of cross adaptation to the actual ambient temperature or show that prenatal temperature possibly have no long-lasting influences.

Also neuronal hypothalamic c-Fos expression, which indicates activation of numerous neurons at one time, shows a stepwise development during embryonic and post hatching ontogeny, similar with the electrophysiological results obtained from single neuronal activity. The results of our previous study on different incubated chicken embryos indicate that acute heat stress on the last day of incubation led to a clear expression of c-Fos in the $\mathrm{PO} / \mathrm{AH}$ of all investigated embryos when compared to the unstressed control [23]. Although, the differences in c-Fos expression between the warm and cold incubated embryos as well as the group incubated at $37.5^{\circ} \mathrm{C}$ were not significant because of the high individual variation; the trend in this study was that warm as well as cold temperature-experienced chick embryos have a lower neuronal c-Fos expression than the inexperienced control group. These results were in accordance with our results of single cell recordings of hypothalamic neurons in late term Muscovy duck embryos [34], which were described above. In our actual study, in the 4 weeks old chicken no differences in c-Fos expression could be found between different incubated chickens, which is similar with results we obtained from recordings of single neurons in the hypothalamus of 4 weeks old Muscovy ducks (unpublished own results). It is a sign of a new step in the reorganization of the neuronal network controlling body temperature, which could be related to the normal change from the juvenile to the adult type of neuronal hypothalamic thermosensitivity, but also by cross adaptation to the actual post hatching environment. Finally, in 8 weeks old chickens significant differences in acute heat induced neuronal hypothalamic cFos expression between the cold -and warm incubated group were found, characterized by a decreased neuronal c-Fos expression in the cold incubated group and an increased neuronal c-Fos expression in the warm incubated group compared with the level of the regular incubated group.

\section{CONCLUSION}

In conclusion, the opposite effect in short-term heat induced neuronal c-Fos expression between prenatal warm and cold experienced 8 weeks old chickens indicates longlasting modification in cellular and molecular neuronal mechanisms of temperature regulation, which were induced by prenatal environmental influences, viz. changes in incubation temperature, during a critical period in the development of the thermoregulatory system. The long-lasting reorganization of this network occurs stepwise and can be influenced by postnatal cross adaptation to the actual climatic conditions. Demonstrated by neuronal c-Fos expression, the incubation temperature induced developmental pattern of the hypothalamic neuronal network, which is involved in thermoregulation, is similar with the pattern, which we found in a previous study using extracellular recordings of single neurons activity, to a higher extent. Correlations between both parameters, which are involved in neuronal plasticity, can be accepted and were described in other publications [38, 39], too.

\section{ABBREVIATIONS \\ $\mathrm{DAB}=$ Diaminobenzidine tetrahydrochloride \\ IEG $=$ Immediate-early genes \\ $\mathrm{Pb}=$ Phosphate buffer \\ $\mathrm{PO} / \mathrm{AH}=$ Preoptical area of the anterior hypothalamus}

\section{ACKNOWLEGEMENT}

The actual study was supported by the Deutsche Forschungsgemeinschaft (JA 1440/1-1).

\section{REFERENCES}

[1] Tzschentke B, Plagemann A. Imprinting and critical periods in early development. World's Poult Sci J 2006; 62: 626-37.

[2] Tzschentke B. Attainment of thermoregulation as affected by environmental factors. Poult Sci 2007; 86: 1025-36.

[3] Tzschentke B. Monitoring the development of thermoregulation in poultry embryos and its influence by incubation temperature. Comput Electron Agric 2008; 64: 61-71.

[4] Minne B, Decuypere E. Effects of late prenatal temperatures on some thermoregulatory aspects in young chickens. Arch Exp Vet Med 1984; 38: 374-83.

[5] Tzschentke B, Nichelmann M. Development of avian thermoregulatory system during the early postnatal period: development of the thermoregulatory set-point. Ornis Fen 1999; 76: 189-98. 
[6] Tzschentke B, Basta D. Early development of neuronal hypothalamic thermosensitivity in birds: influence of epigenetic temperature adaptation. Comp Biochem Physiol A 2002; 131: 82532.

[7] Miyata S, Ishiyama M, Shibata M, Nakashima $T$, Kiyohara $T$. Infant cold exposure changes Fos expression to acute cold stimulation in adult hypothalamic brain regions. Neurosci Res 1998; 31: 219-25.

[8] Morgan JI, Curran T. Proto-oncogene transcription factors and epilepsy. Trends Pharmacol Sci 1991; 12: 343-9.

[9] Kanitz E, Puppe B, Tuchscherer M, Heberer M, Viergutz T, Tuchscherer A. A single exposure to social isolation in domestic piglets activates behavioural arousal, neuroendocrine stress hormones, and stress-related gene expression in the brain. Physiol Behav 2009; 98(1-2): 176-85.

[10] Harikai N, Tomogane K, Sugawara T, Tashiro S. Differences in hypothalamic Fos expression between two heat stress conditions in conscious mice. Brain Res Bull 2003; 61: 617-26.

[11] Yoshida K, Maruyama M, Hosono T, et al. Fos expression induced by warming the preoptic area in rats. Brain Res 2002; 933: 109-17.

[12] Hildebrandt JP, Gerstberger R, Schwarz M. In vivo and in vitro induction of c-fos in avian exocrine salt gland cells. Am J Physiol 1998; 275: C951-7.

[13] Robertson HA. Immediate-early genes, neuronal plasticity, and memory. Biochem Cell Biol 1992; 70: 729-37.

[14] Kaczmarek L. Molecular biology of vertebrate learning: is c-Fos a new beginning? J Neurosci Res 1993; 34: 377-81.

[15] Dragunow M. A role for immediate-early transcription factors in learning and memory. Behav Genet 1996; 26: 293-9.

[16] Tischmeyer W, Grimm R. Activation of immediate early genes and memory formation. Cell Mol Life Sci 1999; 55: 564-74.

[17] Sanyal S, Sandstrom DJ, Hoeffer CA, Ramaswami M. AP-1 functions upstream of CREB to control synaptic plasticity in drosophila. Nature 2002; 416: 870-4.

[18] Katche C, Bekinschtein P, Slipczuk L, et al. Delayed wave of c-Fos expression in the dorsal hippocampus involved specifically in persistence of long-term memory storage. Proc Natl Acad Sci USA 2010; 107(1): 349-54.

[19] Rose SP. How chicks make memories: the cellular cascade from cFos to dendritic remodelling. Trends Neurosci 1991; 14: 390-7.

[20] Mileusnic R, Anokhin K, Rose SPR. Antisense oligodeoxynucleotides to c-Fos are amnestic for passive avoidance in the chick. NeuroReport 1996; 7: 1269-72.

[21] Yanagihara S, Yagi T, Matsushima T. Distinct mechanisms for expression of Fos-like immunoreactivity and synaptic potentiation in telencephalic hyperstriatum of the quail chick. Brain Res 1998; 779: 240-53.

[22] Yahav S, McMurtry JP. Thermotolerance acquisition in broiler chickens by temperature conditioning early in life - the effect of timing and ambient temperature. Poult Sci 2001; 80: 1662-6.

[23] Janke O, Tzschentke B. Hypothalamic c-Fos expression of temperature experienced chicken embryos after acute heat exposure. In: Yahav S, Tzschentke B, Eds. New insights into fundamental physiology and peri-natal adaptation of domestic Fowl. Nottingham University Press: UK 2006; pp. 109-15.

[24] Kuenzel WJ, Masson M. A stereotaxic atlas of the brain of the chick (Gallus domesticus). The John Hopkins University Press: Baltimore 1988.
[25] Kuenzel WJ, van Tienhoven A. Nomenclature and location of avian hypothalamic nuclei and associated circumventricular organs. J Comp Neurol 1982; 206: 293-313.

[26] Abraham WC, Demmer J, Williams JM, et al. Correlations between immediate early gene induction and the persistence of long-term potentiation. Neuroscience 1993; 56(3): 717-27.

[27] Worley PF, Bhat RV, Baraban JM, Erickson CA, McNaughton BL, Barnes CA. Thresholds for synaptic activation of transcription factors in hippocampus: correlation with long-term enhancement. J Neurosci 1993; 13(11): 4776-86.

[28] Labunskay G, Meiri N. R-Ras3/(M-Ras) is involved in thermal adaptation in the critical period of thermal control establishment. J Neurobiol 2006; 66(1): 56-70.

[29] Katz A, Meiri N. Brain-derived neurotrophic factor is critically involved in thermal-experience-dependent developmental plasticity. J Neurosci 2006; 26(15): 3899-907.

[30] Yossifoff M, Kisliouk T, Meiri N. Dynamic changes in DNA methylation during thermal control establishment affect CREB binding to the brain-derived neurotrophic factor promoter. Eur $\mathrm{J}$ Neurosci 2008; 28(11): 2267-77.

[31] Kisliouk T, Meiri N. A critical role for dynamic changes in histone H3 methylation at the Bdnf promoter during postnatal thermotolerance acquisition. Eur J Neurosci 2009; 30(10): 1909-22.

[32] Kisliouk T, Ziv M, Meiri N. Epigenetic control of translation regulation: alterations in histone $\mathrm{H} 3$ lysine 9 post-translation modifications are correlated with the expression of the translation initiation factor 2B (Eif2b5) during thermal control establishment. Dev Neurobiol 2010; 70(2): 100-13.

[33] Tzschentke B, Basta D. Development of hypothalamic neuronal thermosensitivity in birds during the perinatal period. $\mathrm{J}$ Therm Biol 2000; 25: 119-23.

[34] Loh B, Maier I, Winar A, Janke O, Tzschentke B. Prenatal development of epigenetic adaptation processes in poultry: changes in metabolic and neuronal thermoregulatory mechanisms. Avian Poult Biol Rev 2004; 15 (3/4): 119-28.

[35] Tzschentke B, Basta D, Janke O, Maier I. Characteristics of early development of body functions and epigenetic adaptation to the environment in poultry: focused on development of central nervous mechanisms. Avian Poult Biol Rev 2004; 15: 107-18.

[36] Sallagundala N, Yakimova K, Tzschentke B. Characterization of neuronal hypothalamic plasticity in chicken: a comparative analysis. In: Yahav S, Tzschentke B, Eds. New insights into fundamental physiology and peri-natal adaptation of domestic fowl. Nottingham University Press: UK 2006, pp. 99-108.

[37] Nakashima T, Pierau F-K, Simon E, Hori T. Comparison between hypothalamic thermoresponsive neurons from duck and rat slices. Pfluegers Arch 1987; 409: 236-43.

[38] Svarnik OE, Alexandrov YI, Gavrilov VV, Grinchenko YV, Anokhin KV. Fos expression and task-related neuronal activity in rat cerebral cortex after instrumental learning. Neuroscience 2005; 136(1): 33-42.

[39] Mayer U, Watanabe S, Bischof H-J. Hippocampal activation of immediate early genes Zenk and c-Fos in zebra finches (Taenioygia guttata) during learning and recall of a spatial memory task. Neurobiol Learn Mem 2010; 93(3): 322-9. 